\title{
The change of therapeutic trends in the thymic epithelial tumor
}

\author{
Chang Hyun Kang \\ Department of Thoracic and Cardiovascular Surgery, Seoul National University Hospital, Seoul National University College of Medicine, Seoul, \\ South Korea \\ Correspondence to: Chang Hyun Kang, MD, PhD. Department of Thoracic and Cardiovascular Surgery, Seoul National University Hospital, 101 \\ Daehak-ro, Chongro-gu, Seoul 03080, South Korea. Email: chkang@snu.ac.kr. \\ Provenance: This is an invited article commissioned by the Section Editor Laura Chiara Guglielmetti (Cantonal Hospital Winterthur, Kantonsspital \\ Winterthur, Switzerland). \\ Comment on: Ruffini E, Guerrera F, Brunelli A, et al. Report from the European Society of Thoracic Surgeons prospective thymic database 2017: a \\ powerful resource for a collaborative global effort to manage thymic tumours. Eur J Cardiothorac Surg 2019;55:601-9.
}

Submitted Oct 05, 2019. Accepted for publication Oct 21, 2019.

doi: $10.21037 /$ jtd.2019.11.15

View this article at: http://dx.doi.org/10.21037/jtd.2019.11.15

Thymic epithelial tumor (TET) is a unique and rare malignant tumor with low incidence. The tumor shows different prognosis ranging from indolent to highly aggressive feature. A biopsy is usually not recommended if surgical treatment is expected because of the high prevalence of tumor dissemination through the biopsy tract. Therefore upfront surgery without pathologic diagnosis is common practice, especially in early-stage TET. Therefore, there is a relatively high need for minimally invasive thymectomy (MIT) because the operation should be performed in the setting of uncertain diagnosis. In advanced TET, the tumor occasionally invades vital structures including great vessels or heart, which increases the risk of incomplete resection. Pleural metastasis is also common in advanced TET. Therefore systemic treatment is an important issue in TET.

The paper written by Ruffini and colleagues (1) reported current trend of surgical treatment patterns in TET. They analyzed 1,122 patients over ten years in the European Society Thoracic Surgeons (ESTS) prospective database. Three significant findings of the paper were (I) increase of thymic carcinoma, (II) increase of MIT, and (III) higher adaption of perioperative systemic treatment. In my opinion, the authors correctly pointed out the current changing trend in the treatment of TET and suggested the implication on future studies.

In the paper, the authors could not suggest a clear answer to why the prevalence of thymic carcinoma increased recently. They reported the incidence of thymic carcinoma was $28 \%$, which was higher than the historical studies or the past ESTS database study. They suggested geographic distribution, dedicated referral or improved pathologic diagnosis might be the causes of the change. I think each factor could be a possible cause of the change. However, I want to add two additional factors that should be considered. The first one is the aging of the patients. We already know we are experiencing elderly patients more and more and increasing age of surgical patients. Our group reported that TET is more prevalent in elderly patients than the younger population (2) and the mean age of thymic carcinoma is higher than that of thymoma (3). Aging trend of the patients may be one possible explanation for the increase of thymic carcinoma in my opinion. The second probable cause is the advancement of multimodality treatment. Because thymic carcinoma occasionally presents as an advanced tumor at the initial presentation, resectability is questioned in many cases. Neoadjuvant treatment can downstage the tumor and potentially increase the number of surgical cases. Several studies reported neoadjuvant treatment increased resectability in the advanced TET (4).

Ruffini and colleagues also reported the increasing trend of MIT in the ESTS database. The increase of MIT is a global trend in the treatment of TET. In the past ESTS database study, only $6 \%$ of the patients received MIT, however it increased up to $33 \%$ in a recent prospective database (thoracoscopic surgery in $20 \%$ and robotic surgery in $13 \%)$. It is well known that sternotomy increases bleeding, pain and complication postoperatively. Many 
studies emphasized this issue and MIT could successfully decrease postoperative adverse events and improve early outcomes $(5,6)$. Although there has been no randomized controlled study comparing open thymectomy and MIT, it is a general consensus that the early result of MIT is better than open thymectomy. However, there are also unsolved issues about the completeness of thymectomy and long-term outcomes in MIT. In real clinical practice, many surgeons perform thymomectomy rather than total thymectomy when conducting MIT, because complete removal of contralateral thymic tissue and upper cervical poles are technically demanding. For a long time, total thymectomy has been a gold standard in the treatment of TET to prevent local recurrence and complete remission of myasthenia gravis. However, recent studies from JART (7) and KART (8) reported thymomectomy or partial thymectomy is enough for early stage TET. The longterm recurrence-free survival was comparable to total thymectomy. These findings suggest radical thymectomy may not be necessary in the early-stage of TET and more safe application of MIT may be possible in selected patients. However, more studies are necessary to draw a general consensus on this issue.

The increase of systemic treatment was also identified in the study. Neoadjuvant chemotherapy increased from $9 \%$ to $15 \%$ and adjuvant chemotherapy from $2 \%$ to $16 \%$. As I mentioned previously, neoadjuvant chemotherapy has been adapted to down-stage the tumor and increase the complete resection rate (4). It is a promising treatment policy for advanced tumors with marginal resectability. I think it can contribute to the increase of the surgical resection rate. However, whether such multi-modality treatment can improve long-term survival or not is debatable. Our group reported the comparative result between upfront surgery and neoadjuvant chemotherapy followed by surgery using the KART database (9). We could not find any difference in long-term survival between the groups. Although we reported the negative result for neoadjuvant chemotherapy, I think it is too early to conclude that there are no advantages of neoadjuvant chemotherapy in long-term outcomes. The more reliable and robust study, for example, randomized controlled trials, may answer the question. The other direction is to find an effective systemic treatment regimen. The current chemotherapy regimen is based on small studies on palliative chemotherapy with modest response (10). More effective chemotherapy regimens are necessary to treat the advanced TET or new agent, for example, a target agent or immunotherapy may be a promising alternative treatment option.

The study written by Ruffini and colleagues reported current changing trend in the treatment of TET. It is timely and offers many suggestions. Like many other medical areas, we are facing new changes in the area of TET. The patients are changing, surgical techniques are developing, and new systemic agents are being introduced. Hopefully I am expecting further researches which can resolve several issues raised in this article in the near future.

\section{Acknowledgments}

None.

\section{Footnote}

Conflicts of Interest: The author has no conflicts of interest to declare.

Ethical Statement: The author is accountable for all aspects of the work in ensuring that questions related to the accuracy or integrity of any part of the work are appropriately investigated and resolved.

\section{References}

1. Ruffini E, Guerrera F, Brunelli A, et al. Report from the European Society of Thoracic Surgeons prospective thymic database 2017: a powerful resource for a collaborative global effort to manage thymic tumours. Eur J Cardiothorac Surg 2019;55:601-9.

2. Yoon $\mathrm{SH}$, Choi $\mathrm{SH}$, Kang $\mathrm{CH}$, et al. Incidental Anterior Mediastinal Nodular Lesions on Chest CT in Asymptomatic Subjects. J Thorac Oncol 2018;13:359-66.

3. Hwang Y, Kang CH, Park S, et al. Impact of Lymph Node Dissection on Thymic Malignancies: Multi-Institutional Propensity Score Matched Analysis. J Thorac Oncol 2018;13:1949-57.

4. Ahmad U, Huang J. Induction Therapy for Thymoma. Thorac Surg Clin 2016;26:325-32.

5. Kang CH, Hwang Y, Lee HJ, et al. Robotic Thymectomy in Anterior Mediastinal Mass: Propensity Score Matching Study With Transsternal Thymectomy. Ann Thorac Surg 2016;102:895-901.

6. Friedant AJ, Handorf EA, Su S, et al. Minimally Invasive versus Open Thymectomy for Thymic Malignancies: Systematic Review and Meta-Analysis. J Thorac Oncol 2016;11:30-8. 
7. Nakagawa K, Yokoi K, Nakajima J, et al. Is Thymomectomy Alone Appropriate for Stage I (T1N0M0) Thymoma? Results of a Propensity-Score Analysis. Ann Thorac Surg 2016;101:520-6.

8. Narm KS, Lee CY, Do YW, et al. Limited thymectomy as a potential alternative treatment option for early-stage thymoma: A multi-institutional propensity-matched study.
Lung Cancer 2016;101:22-7.

9. Park S, Park IK, Kim YT, et al. Comparison of Neoadjuvant Chemotherapy Followed by Surgery to Upfront Surgery for Thymic Malignancy. Ann Thorac Surg 2019;107:355-62.

10. Schmitt J, Loehrer PJ Sr. The role of chemotherapy in advanced thymoma. J Thorac Oncol 2010;5:S357-60.

Cite this article as: Kang $\mathrm{CH}$. The change of therapeutic trends in the thymic epithelial tumor. J Thorac Dis 2019;11(12):5652-5654. doi: 10.21037/jtd.2019.11.15 